\title{
Verification Study of a CFD-RANS Code for Turbulent Flow at High Reynolds Numbers
}

\author{
Omer Musa, Zhou Changshen, Chen Xiong, and Li Yingkun
}

\begin{abstract}
This article surveys the verification and validation methods of the in-house codes. A practical aspect of verification and validation procedure of an in-house code is provided. In order to solve Reynolds-averaged Navier-Stokes (RANS) equations of the compressible flow field, a computational fluid dynamics (CFD) computer program is developed. NASA Langley's CFL3D and FUN3D, flow solvers are adopted to verify and validate the developed code. Two test cases are studied; two-dimensional (2-D) zero pressure gradient flat plate and non-zero pressure gradient 2-D NACA 0012 airfoil, each which uses 5 systematically refined meshes. First, the code and solution verification for both cases are investigated. The observed order of accuracy and numerical uncertainty results agree with those of CFL3D, FUN3D, Cobalt, and RavenCFD flow solvers. For model validation, an experimental data is used as well. The predicted skin friction coefficient over the turbulent flat plate surface shows good agreement with the experiment and with the flow solvers. Furthermore, skin friction, pressure, drag, and lift coefficients for the 2-D NACA 0012 airfoil at three angles of attack $\left(\alpha=0^{\circ}, \alpha=10^{\circ}\right.$, and $\left.\alpha=15^{\circ}\right)$ are estimated. The results agree well. The presented evidences indicate that the code is verified and validated correctly.
\end{abstract}

Index Terms-Verification, computational fluid dynamics, AUSMPW, SST turbulence model, numerical uncertainty.

\section{INTRODUCTION}

Researchers have paid much attention on computational fluid dynamics (CFD) during decades. Contemporary, CFD codes and the commercial software's are becoming one of the most important parts of real projects, allowing experimental tests to be conducted only in the final stages of the project. However, computer speed, shape complexity, flow behavior, meshing strategy and other factors are restricting the accuracy of predicted results. To simulate the flow around objects (or moving objects), flow with separation, and combustion simulation, some hypothetical models exist to deal with turbulence, Reynolds-averaged Navier-Stokes (RANS) based turbulence model are widely used one. Code, solution verification, and model validation are the second challenge facing developers after writing codes, which are; 1) determining the code's observed order of accuracy, as compared with its formal order, 2) quantify numerical accuracy of code's predicted solutions for verifying codes, and 3) assess how accurately the model represents the physical flow by comparing the results with experimental results, for validating codes.

Manuscript received December 7, 2015; revised February 2016.

The authors are with the School of Mechanical Engineering, Nanjing University of Science and Technology, Nanjing, China (e-mail: omer.musa1@hotmail.com).
In general, the words verification and validation are synonymous; however, in engineering usage, they have very different meanings. The characterization of the numerical approximation errors associated with a simulation is called verification [1]. In other words, verification is concerned with whether the code is solving the chosen equations correctly and error-free while the validation deals with physics and addresses the appropriateness of the model [2].

There are two concepts of verification; the first one is the code verification, which is the process ensuring, to the degree possible, that bug-free and providing that systematic mesh refinement results in improved observed order of accuracy. The second one is the solution verification, which is the process of qualifying the numerical errors that occur in the numerical simulation. These numerical errors are, round-off error, iterative error, discretization error, and errors due to computer programming mistakes [2], [3].

The methods that can be used for code verification are, 1) Method of exact solution, this approach compares the numerical solution with the exact solution to the governing equations [4]. 2) Method of manufactured solution, this approach manufactures an exact solution to a modified set of equation rather than finding the governing equations exact solution [4]-[7]. 3) Using of benchmark solutions which have been verified [4]. 4) Code-to-code comparison, where the results from unverified code are compared to the results of verified code [8].

Numerical errors always exist when partial differential equations are solved numerically, solution verification deals with the assessment of these errors. There are three main concepts of solution verification; 1) input data verification, which is ensuring that the correct input files, grids, tabulated data, etc. 2) output data verification, which is ensuring that the user takes the correct post-processing steps after the simulation in completed. 3) Numerical errors estimation (discretization error, iterative convergence error, and round-off error) [4]. Roy and William [2] have proposed study included new concepts for estimating uncertainties due to numerical errors. Roy [4], [9] has carried out investigation of code and solution verification methods and estimating discretization error.

Recently, Jacob and Roy [3] conducted an extensive verification and validation study on two commercial solvers, Cobalt and RavenCFD, for low subsonic 2-D applications (turbulent flat plate and NACA 0012 airfoil at $\alpha=0^{\circ}$ ), to verify the turbulence models. They found that the observed order of accuracy of these solvers between 1 and 2, and the turbulence models were implemented correctly. Oisin and Aaron [10] have examined the turbulent flow validation of the Helios Strand flow solver for different flow cases, 
including the same benchmark cases used by Jacob and Roy [3] (turbulent flat plate and NACA 0012 airfoil at $\alpha=0^{\circ}$ ). Their results match with NASA Langley solvers (CFL3D and FUN3D), for Spalart-Allmaras (S-A) turbulence model. Atsushi and Mitsuhiro [11] conducted validation study on FaSTAR and UPACS flow solvers for the same benchmark cases using S-A turbulence model. Finally, Cambier and Vincent [12] have carried out verification and validation study of the Onera elsA flow solver for turbulent flow over the 2-D zero pressure gradient flat plate and 2-D backward facing step, they adopted different turbulence models including SST turbulence model. Most of such detailed verification studies have been done on commercial flow solvers; a few are available for in-house CFD codes. Many of the in-house CFD codes developers concern with validation only, which is not well enough to describe the robust of their codes as mentioned in the literature. Therefore, a detailed study on how to verify the in-house CFD codes have been done to have more attention on importance of verification beside the validation for in-house CFD codes.

The goals of this paper are to 1) provide a review of verification procedures used recently for computational simulations, and 2) verify and validate an in-house code in details to give a practical aspect of verification and validation procedures. The verification and validation have been done by comparing the present code solution and behavior with NASA Langley flow solvers [13], CFL3D (cell-centered structured) and FUN3D (node-centered unstructured), along with the study conducted by Jacob and Roy [3] on Cobalt and RavenCFD flow solvers. Experimental data are used to deal with the validation issue [14], [15].

The present code will be used as a base code for future expansion of combustion code to simulate 2-D turbulent combustion flow in ramjet engine. The benchmark cases have been used are the same as cases used in literature; 2-D zero pressure gradient flat plate and NACA 0012 airfoil at three angles of attack $\left(\alpha=0^{\circ}, \alpha=10^{\circ}\right.$, and $\left.\alpha=15^{\circ}\right)$, at high Reynolds number and subsonic flow.

\section{GOVERNING EQUATIONS}

\section{A. RANS Equations}

In order for fluid flow to describe the exchange (flux) of mass, momentum and energy through the boundary surface $S$ of a control volume $\Omega$, which is fixed in space, the two-dimensional and/or axisymmetric Reynolds-averaged Navier-Stokes equations in integral form are employed [16].

$$
\begin{aligned}
& \frac{\partial}{\partial t} \iiint_{\Omega} U d \Omega+\iint_{S}\left(F \cdot n_{x}+G \cdot n_{y}\right) d S \\
& -\iint_{S}\left(F_{v} \cdot n_{x}+G_{v} \cdot n_{y}\right) d S=\iiint_{\Omega}\left(\boldsymbol{H}+\boldsymbol{H}_{v}\right) \cdot \varepsilon d \Omega .
\end{aligned}
$$

where $n=n_{x} i+n_{y} j$ is the unit normal vector in outward direction of $S$; $t$ is the time; $\varepsilon=0$ for two-dimensional plane flow, and $\varepsilon=1$ for axisymmetric flow; $U, F, G$, and $H$ are conservative vector, convective flux vector, viscous flux vector, and axisymmetric source term vector, respectively.

$$
\begin{gathered}
U=\left\lfloor\begin{array}{c}
\rho \\
\rho u \\
\rho v \\
E
\end{array}\right\rfloor, F=\left\lfloor\begin{array}{c}
\rho u \\
\rho u^{2}+p \\
\rho u v \\
(E+p) u
\end{array} \mid, G=\left\lfloor\begin{array}{c}
\rho v \\
\rho v u \\
\rho v^{2}+p \\
(E+p) v
\end{array} \mid,\right.\right. \\
F_{v}=\left\lfloor\begin{array}{c}
0 \\
0 \\
\tau_{x x} \\
\tau_{x y} \\
u \tau_{x x}+v \tau_{x y}+q_{x}
\end{array}\right\rfloor, G_{v}=\left\lfloor\begin{array}{c}
\tau_{y y} \\
u \tau_{y x}+v \tau_{y y}+q_{y}
\end{array}\right\rfloor, \\
0 \\
H v \\
\quad=\frac{1}{y}\left\lfloor\begin{array}{c}
\rho v u \\
\rho v^{2} \\
(E+p) v
\end{array} \mid, H_{v}=\frac{1}{y}\left\lfloor\begin{array}{c}
\tau_{y x}-\tau_{\theta \theta} \\
u \tau_{y x}+v \tau_{y y}+q_{y}
\end{array}\right\rfloor .\right.
\end{gathered}
$$

with

$$
\begin{gathered}
\tau_{x x}=\frac{2}{3} \mu\left(2 \frac{\partial u}{\partial x}-\frac{\partial v}{\partial y}\right), \tau_{y y}=\frac{2}{3} \mu\left(2 \frac{\partial v}{\partial y}-\frac{\partial u}{\partial x}\right), q_{x}=k \frac{\partial T}{\partial x}, \\
\tau_{x y}=\tau_{y x}=\mu\left(\frac{\partial u}{\partial y}+\frac{\partial v}{\partial x}\right), q_{y}=k \frac{\partial T}{\partial y}, E=\frac{p}{\gamma-1}+\frac{1}{2} \rho\left(u^{2}+v^{2}\right),
\end{gathered}
$$

where $\rho, p, T, u, v$, and $E$ represent the density, pressure, temperature, $\mathrm{x}$-velocity, $\mathrm{y}$-velocity, and total energy, respectively. The variable $\tau$ is the shear stress, $k$ is the thermal conductivity coefficient of the fluid, $\gamma$ is the ratio of specific heats, and $\mu$ is the total effective viscosity which equals to the summation of laminar and turbulent viscosities.

\section{B. The SST Turbulence Model}

The turbulence model used to close the system of equations is the Menter's shear stress transport (SST) turbulence model Menter [17]. The basic foundation of the Menter's turbulence model was first proposed by Wilcox [18], which works by solving a turbulent/frequency based model. Wilcox model is known as the original k- $\omega$ model. The use of $k-\omega$ model in the inner regions of the boundary layer makes the formulation usable through the viscous sub-layer. To avoid the common problem of $k$ - $\omega$ model that the model is too sensitive to the inlet free-stream turbulence properties [19], the SST model switches to $k-\varepsilon$ [20] model in the free-stream. However, $k-\varepsilon$ model was not able to capture accurately the turbulence of turbulent boundary layers due to separation [21]. Therefore, the SST model uses $k$ - $\omega$ model at the walls and $k-\varepsilon$ model away from walls by using the blending factor to ensure a smooth transition between the two models.

\section{NumericAl SOLUTION METHOD}

\section{A. AUSMPW Scheme}

In this code, viscous terms are treated by using a second-order centered scheme to evaluate the diffusive fluxes, and inviscid terms are treated by using advection upstream splitting method by pressure-based weight function (AUSMPW) to determine the numerical convective flux at cell faces, which is designed to remove the oscillations of AUSM+ near the wall or across a strong shock by using pressure-based weight functions [22]. 


\section{B. Third-Order MUSCL}

In general, upwind schemes have a first-order spatial accuracy. Therefore, to improve the spatial accuracy, a third-order monotone upstream centered scheme for conservation laws (MUSCL) approach [23] with Van Albada limiter [24] is implemented to avoid non-physical shock discontinuities, which is interpolate higher order left and right states across a cell interface.

\section{Time Method}

For time discretization, the semi-discretized Reynolds-averaged Navier-Stokes equation can be written as

$$
\frac{\partial U}{\partial t}=R(U)
$$

where $R(U)$ is a discretization of the spatial operator. Equation (2) is integrated by a method-of-lines approach using the third-order three-stage TVD Runge-Kutta method [25] which reads

$$
\begin{aligned}
& U^{(1)}=U^{(n)}+\Delta t R\left(U^{(n)}\right) \\
& U^{(2)}=\frac{3}{4} U^{(n)}+\frac{3}{4} U^{(1)}+\frac{1}{4} \Delta t R\left(U^{(1)}\right) \\
& U^{(\mathrm{n}+1)}=\frac{1}{3} U^{(n)}+\frac{2}{3} U^{(2)}+\frac{2}{3} \Delta t R\left(U^{(2)}\right) .
\end{aligned}
$$

where $\Delta t$ is the time step and $n$ is the time level.

\section{Initial and Boundary Conditions}

In addition to the governing equations, the boundary conditions and initial conditions are necessary to fully define the flow field. The boundary conditions have been used in the present code are; 1) no-slip adiabatic wall boundary condition, 2) far field boundary condition, 3) symmetry boundary condition, 4) inflow boundary condition, and 5) outflow boundary condition. Riemann invariants corresponding to the incoming and outgoing waves are implemented [26]. Appropriate boundary conditions are applied using ghost cells around the main grid. The free-stream variables are used as initial conditions to prescribe the flow field variables over the entire domain at initial time. The ambient free-stream turbulence values recommended by Spalart and Rumsey [27] are implemented.

\section{CASE DESCRIPTION}

The present simulation program has been verified and validated using two of the most popular benchmark problems, a 2-D flat plate to verify and validate boundary layer and a 2-D airfoil for non-zero pressure gradient modeling, which are provided by NASA Langley [13].

Fig. 1 illustrates the 2-D flat plate setup from NASA Langley [13], which consists of five levels of systematically refined structured meshes, with the average $y^{+}$values for the first cell range between 0.1 for the finest grid to 1.7 for the coarsest. The plate leading edge begins at $x=0.0$ and extends to $x=2.0$. Free-stream Mach number, $M_{\infty}=0.2$. Reynolds number is $R e=5 \times 10^{6}$, based on a plate length. The reference temperature is $T_{r e f}=300 \mathrm{~K}$. The inflow total pressure and total temperature are $P_{t} / P_{r e f}=1.02828$ and $T_{t} / T_{r e f}=1.008$, respectively. At the outflow boundary, static pressure is $\mathrm{P} / \mathrm{P}_{\text {ref }}$ $=1$ and the other variables are extrapolated from domain interior. A short symmetry boundary condition entry way beginning at $x=-0.33$ is used to allow for proper inflow condition. No-slip adiabatic wall condition is applied for the wall, for the upper boundary, symmetry boundary condition is employed. $\mathrm{y}^{+}$is defined as

$$
y^{+}=\frac{y}{v} \sqrt{\tau_{w} / \rho} .
$$

where $y$ is the height of the first cell, $v$ is kinematic viscosity, and $\tau_{w}$ is wall shear stress.

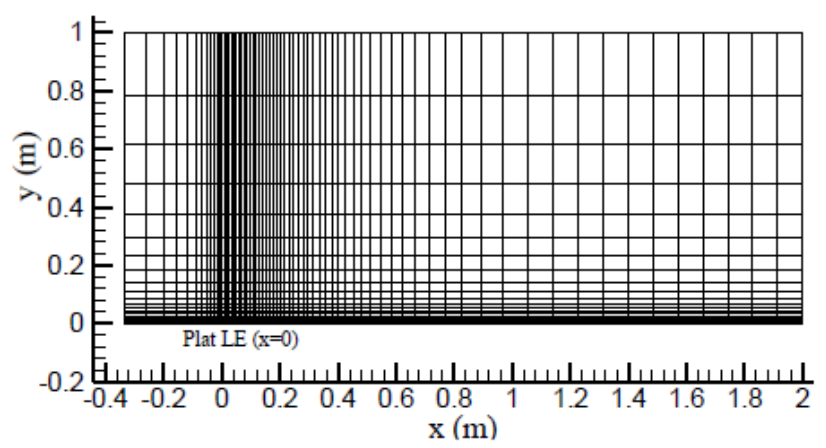

Fig. 1. Turbulent flat plate, the coarsest grid, $35 \times 25$.

Fig. 2 shows the 2-D NACA 0012 airfoil of NASA Langley Research Center [13], which includes five levels of systematically refined C-grid topology structured meshes. The average $y^{+}$values for the first cell range between 0.048 for the finest grid to 1.19 for the coarsest. Fig. 2(a) and (b) show the coarsest grid mesh and close view of 2nd finest grid, respectively. The Mach number, $M_{\infty}=0.15$. Reynolds number is $\mathrm{Re}=6 \times 10^{6}$, based on the chord. For the far field boundaries, the Riemann boundary condition is employed. The reference temperature at far field boundaries is $T_{r e f}=300 \mathrm{~K}$. No-slip adiabatic wall condition is applied through airfoil surface. Three angles of attack are evaluated as $\alpha=0^{\circ}$ for verification study and $\alpha=10^{\circ}, 15^{\circ}$ for validation.

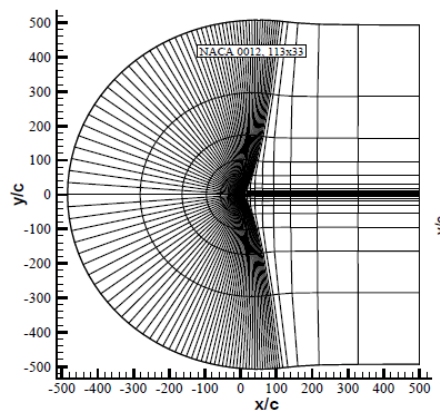

(a)

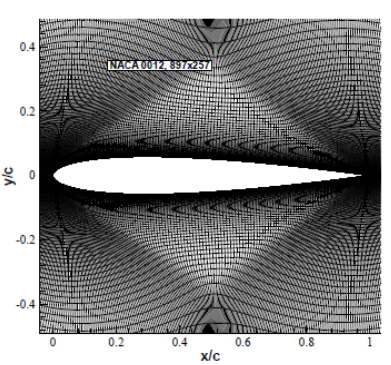

(b)
Fig. 2. NACA 0012 airfoil case, (a) Far view of coarsest grid, 113x33, (b) Close view of 2 nd finest grid, $897 \times 257$.

\section{MODEL VERIFICATION AND VALIDATION}

\section{A. Code Verification}

The best way to verify a code's formal order of accuracy is comparing simulation results with an exact analytical 
solution. Many methods can be used in the absence of the exact solution. Therefore to verify our code, we have adopted two approaches, code-to-code comparison [8], and using of benchmark solutions [4]. For observing order of accuracy, five systematically refined mesh levels are used with grid refinement factor of 2.0 for both cases, which are offered by NASA Langley [13], the observed order of accuracy [4], [9] is achieved by

$$
p=\ln \left(\frac{f_{3}-f_{2}}{f_{2}-f_{1}}\right) / \ln (r) .
$$

where $r$ is a constant grid refinement factor and the variables $f_{1}, f_{2}$, and $f_{3}$ denote solutions from systematically coarser grids, 1 being the finest grid solution. $r=\left(N_{l} / N_{2}\right)^{1 / d}$ for all these grids, where $N$ is the number of cells and $d$ is the dimensionality of the problem ( $d=1$ for $1 \mathrm{D}, d=2$ for $2 \mathrm{D}$ ) [9]. If the observed order of accuracy of interested code tends to the formal order within acceptable range or if the observed order acts similarly to that of a verified code, the code may not be stated unambiguously verified but the model is probably implemented correctly [3].

\section{B. Solution Verification}

The main aspect of the solution verification which is focus on the current study is estimating the numerical errors (discretization error, iterative convergence error, and round-off error) [4].

The numerical errors in computational simulation are; 1) round-off error, which is arises due to the use of finite arithmetic on digital computers. Since we have used double-precision computations, round-off error is too much less than $1 \%$ of the discretization error. 2) Iterative convergence error, which occurs due to incomplete iterative convergence of a discrete system. On other hand, iterative error is the difference between the current iterative solution to the discretized equations and the exact solution to discretized equations. The "machine zero method" is selected to estimate this error for our code, where the residuals are iteratively converged either to machine zero (14 orders of magnitude for double-precision) or to its stabilizing functions (flux limiters and damping), then that converged solution is compared with the current iterative level solution to estimate the iterative error. 3) Discretization error, which is occurs due to the discretization process (the conversion of the differential equations into an algebraic equations). In other words, discretization error is the difference between a numerical solution and the exact solution to the continuum partial differential equations [3], [4].

Equation (8) is used to estimate the uncertainty due to the numerical errors. Iterative errors are assumed to be zero because every solution is converged as far as the scheme allow. Discretization error is estimated by using (7). The exact solution is approximated via Richardson Extrapolation [29], [30]. The basic concept of Richardson Extrapolation is using the discrete solutions on two systematically-refined meshes to estimate the exact solution to the mathematical model, which gives solution more accurate one order than the computed results; e.g., if using a formally first-order method, Richardson Extrapolation approximates a solution that is 2nd-order accuracy. Using $\mathrm{C}_{f}$ as an example, the extrapolated value $\bar{C}_{f, R E}$ is calculated as

$$
\bar{C}_{f, R E}=C_{f}+\frac{\left(C_{f, 1}-C_{f, 2}\right)}{r^{p}-1} .
$$

We then use the extrapolated solution value to estimate the discretization error.

$$
\bar{\varepsilon}_{D E, R E}=\left|C_{f, i}-\bar{C}_{f, R E}\right|,
$$

where $i$ is the respective grid level. Finally, the uncertainty due to numerical error is estimated as

$$
U_{\text {Numerical }}=U_{\text {Dis-error }}+U_{\text {Iter-error }}+U_{\text {Roun-off-error }} \text {. }
$$

with

$$
\begin{aligned}
& U_{\text {Iter-error }}=\left|C_{f, \text { iter-level }}-C_{f, \text { machine-zero }}\right|=0 \\
& U_{\text {Roun-off-error }} \approx 0.01\left(\bar{\varepsilon}_{D E, R E}\right) .
\end{aligned}
$$

The normalization by the fine grid solution in the denominator is omitted for Roache's [30] grid convergence index (GCI) with the modified implementation by Roy [9] to avoid problems when the solution results are near zero, with the safety factor, $F_{s}$, when using more than two systematically refined meshes.

$$
U_{\text {Dis-error }}=\frac{F_{s}}{r^{\tilde{p}}-1}\left|C_{f, 2}-C_{f, 1}\right| .
$$

The original relation suggested by Roache [30] is

$$
U_{\text {Dis-error }}=\frac{F_{s}}{r^{\tilde{p}}-1} \frac{\left|C_{f, 2}-C_{f, 1}\right|}{C_{f, 1}} .
$$

Richardson proposed Richardson extrapolation-based error band $\left(\mathrm{RD} E_{\text {band }}\right)$ to determine the numerical uncertainty, which is agree within $1.5 \%$ of GCI [4].

$$
R D E_{\text {band }}=\frac{F_{s}}{r^{\tilde{p}}-1} \frac{\left|C_{f, 2}-C_{f, 1}\right|}{\bar{C}_{f, \mathrm{RE}}} .
$$

where $F_{s}=1.25$ for $\chi=\left|\left(p-p_{f}\right) / p_{f}\right| \leq 0.1$ or $F_{s}=0.3$ for $\chi>0.1$.

$p_{f}$ is formal order of accuracy, and $p$ is defined in (5), $\tilde{p}=p$ if $\mathrm{F}_{\mathrm{s}}=1.25$, and $\tilde{p}=\min \left(\max (0.1, p), p_{f}\right)$ if $F_{s}=0.3$.

\section{Model Validation}

Validation is assessment of model accuracy by way of comparison of simulation results with experimental measurements [2]. Thus, we used some experimental data offered by NASA Langley [13] and other researchers to validate our model.

\section{RESUlTS AND DiSCUSSION}

\section{A. 2-D Turbulent Flat Plate}

For code verification, Fig. 3 compares the predicted flat plate's drag and skin friction (at $x=0.97008 m$ or $x / L=$ 0.48504 ) coefficients on five systematically refined meshes with NASA Langley results (CFL3D and FUN3D) [13] and 
results obtained by Jacob A. and Roy (Cobalt and RavenCFD) [3]. Dashed horizontal lines mark $\pm 2 \%$ of the average results for the finest grid from NASA benchmark solutions. The grid spacing is normalized by the fine mesh spacing $\left(\Delta x_{1}\right)$. Thus, the grid refinement parameter is defined as $h=\Delta x_{/} \Delta x_{1}$, where $h$ corresponds to the finest grid [4]. On the finest grid, the predicted values of $C_{d}$ and $C_{f}$ are close to those of other four solvers within $1.3 \%$ or less, whereas the four solvers are differing within $0.5 \%$ from each other.

Figs. 4(a) and (b) show the estimated contours for the turbulent eddy viscosity ratios to free-stream viscosity from the present code and FUN3D, respectively. The results and behavior match closely with the benchmark results. Specifically at stream-wise location, $x / L=0.48504$, the predicted eddy viscosity ratio differs from that of FUN3D and Cobalt by less than 5.3\% as shown in Fig. 4(c).

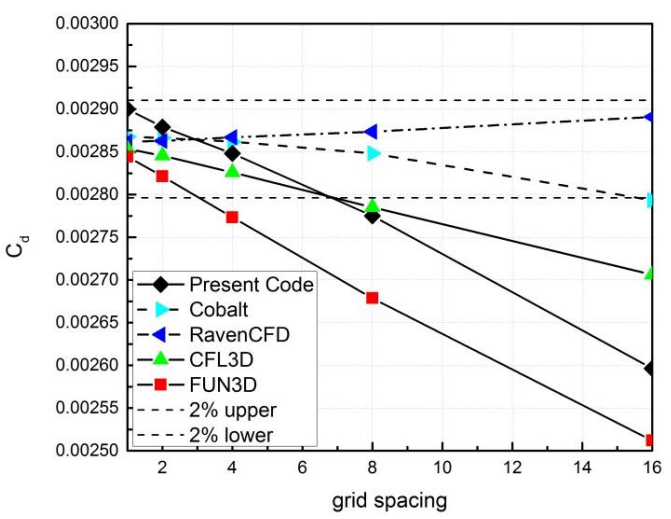

(a) Drag coefficient

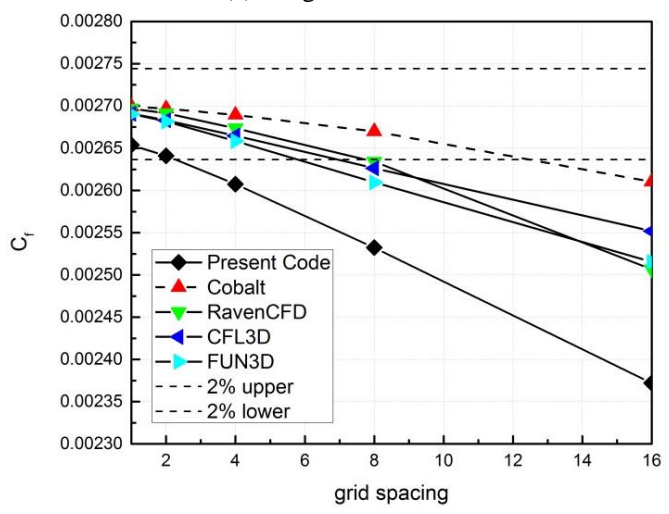

(b) Skin-friction coefficient at $x / L=0.48504$.

Fig. 3. Turbulent flat plate using SST turbulence model on 5 systematically refined meshes.

Figs. 5 and 6 compare non-dimensionalized turbulence kinetic energy solution and energy dissipation rate solution, respectively, on the finest grid with NASA Langley's solvers. As shown, the results are nearly coinciding with those of CFL3D and FUN3D.

For code verification, Table I gives the observed order of accuracy for $C_{d}$ and $C_{f}$, based on the computational results from the three finest grids. For solution verification, table I also shows the total predicted uncertainty due to numerical error using $G C I_{\text {fine }}$ and $R D E_{\text {band }}$. Since the skin friction is less affected by the leading edge singularity, the accuracy of $C_{f}$ generally better than that of $\mathrm{C}_{d}$. The numerical uncertainty (with both $G C I_{\text {fine }}$ and $R D E_{\text {band }}$ ) for the present code is less than $0.4 \%$ for both $\mathrm{C}_{d}$ and $\mathrm{C}_{f}$. Whereas the observed order of accuracy obtained is close to that of FUN3D and slightly different from other solvers. As noted in [3] the formal order of accuracy of other solvers for this case is likely between 1 and 2.

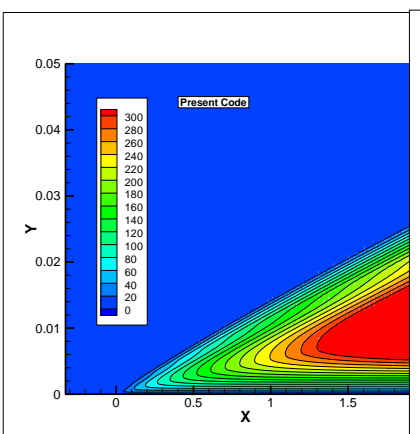

(a)

(c)

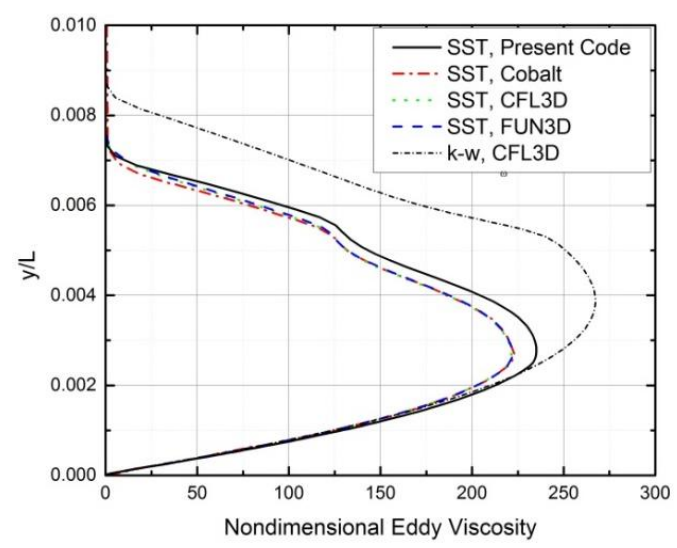

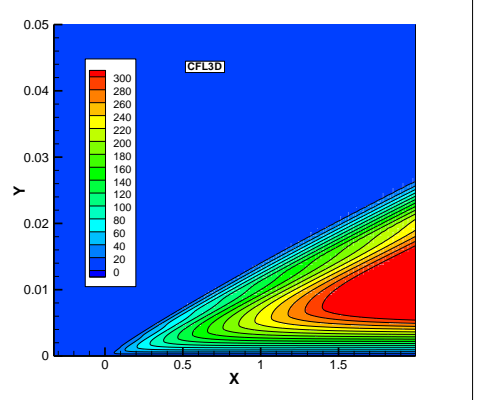

(b)
Fig. 4. Turbulent eddy viscosity ratio $\left(\mu_{t} / \mu_{\infty}\right)$ on flat plate, finest grid.

For validation, Fig. 7 shows the predicted local value for skin friction along the flat plate surface, and the values from CFL3D, FUN3D, Cobalt, RavenCFD, along with experimental results by Wieghart and Tillmann [15]. The estimated result for the finest grid differs from the experiment by less than $5.0 \%$ and by $2.0 \%$ from other solvers. The result of the two measured points close to the leading edge, $x / L<$ 0.05 , differs from experiment by $20 \%$; this may occurred due to interference from the pressure rake in the boundary layer transition region. The other solvers results differ by $5-20 \%$ as noted in [3]. As discussed in [13], agreement suffers at the leading and trailing edges due to the point singularities. It may reasonably be concluded that our result for the turbulent flat plate skin friction is agree well with the results from CFL3D, FUN3D, Cobalt and RavenCFD, along with the experiment for $x / L>0.05$.

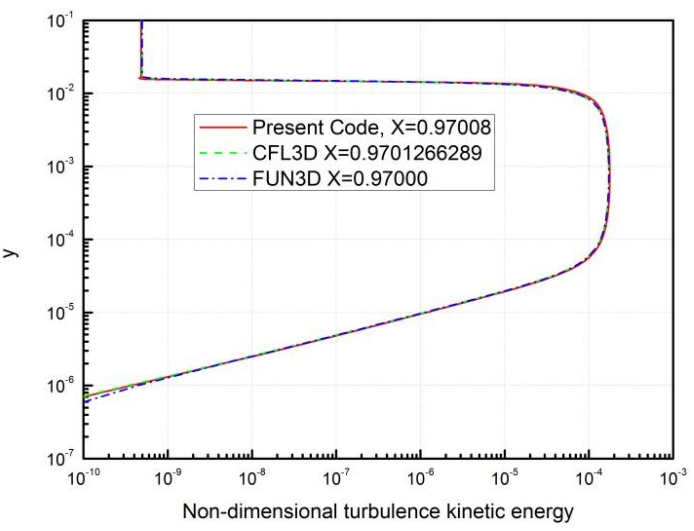

Fig. 5. Non-dimensional turbulence kinetic energy, $k / c_{r e f}^{2}$. 


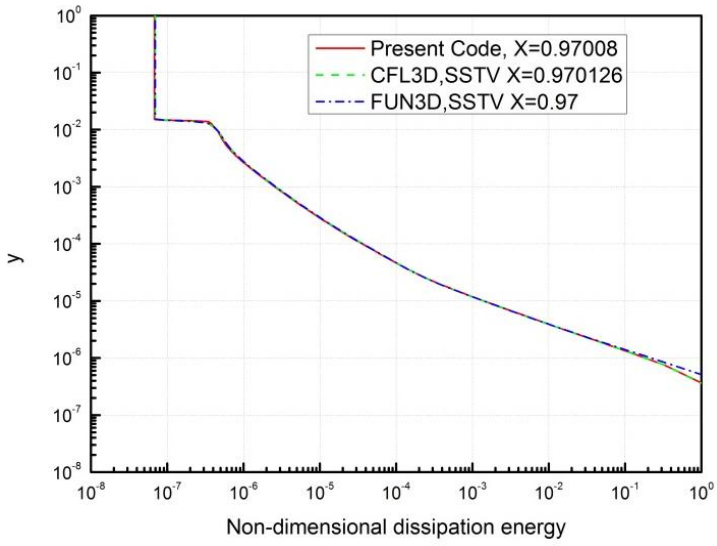

Fig. 6. Non-dimensional energy dissipation rate, $\omega \mu_{\text {ref }} /\left(\rho_{\text {ref }} \mathrm{c}_{\text {ref }}^{2}\right)$.

Fig. 8 compares the stream-wise velocity for two locations downstream on the flat plate with CFL3D and FUN3D results. Good agreement is obtained. Thus, one can conclude that the turbulence model has been correctly implemented for turbulent boundary layer flow in the present code.

TABLE I: TURBUlent Flat Plate

\begin{tabular}{|c|c|c|c|c|c|c|c|}
\hline \multirow{2}{*}{$\begin{array}{l}\text { Turb-ulence } \\
\text { model }\end{array}$} & \multirow{2}{*}{$\begin{array}{l}\text { Flow } \\
\text { solver }\end{array}$} & \multicolumn{3}{|c|}{$C_{d}$} & \multicolumn{3}{|c|}{$C_{f}$ at $x=0.97$} \\
\hline & & $P$ & $\mathrm{GCI}_{\text {Inne }} \%$ & $\mathrm{RDE}_{b a v q} \%$ & $P$ & $\mathrm{GCI}_{f x p} \%$ & $\operatorname{RDE}_{\text {bonn }} \%$ \\
\hline \multirow[t]{3}{*}{ SST } & $\begin{array}{l}\text { Present } \\
\text { Code }\end{array}$ & 1.49 & 0.2617 & 0.2611 & 1.39 & 0.365 & 0.3642 \\
\hline & Cobalt & 1.75 & 0.08 & - & 1.84 & 0.04 & - \\
\hline & RavenCFD & 0.93 & 0.04 & - & 1.60 & 0.13 & - \\
\hline \multirow[t]{2}{*}{ SST-V } & CFL3D & 1.34 & 0.2222 & 0.2218 & 1.21 & 0.277 & 0.2765 \\
\hline & FUN3D & 1.07 & 0.914 & 0.907 & 1.39 & 0.256 & 0.255 \\
\hline
\end{tabular}

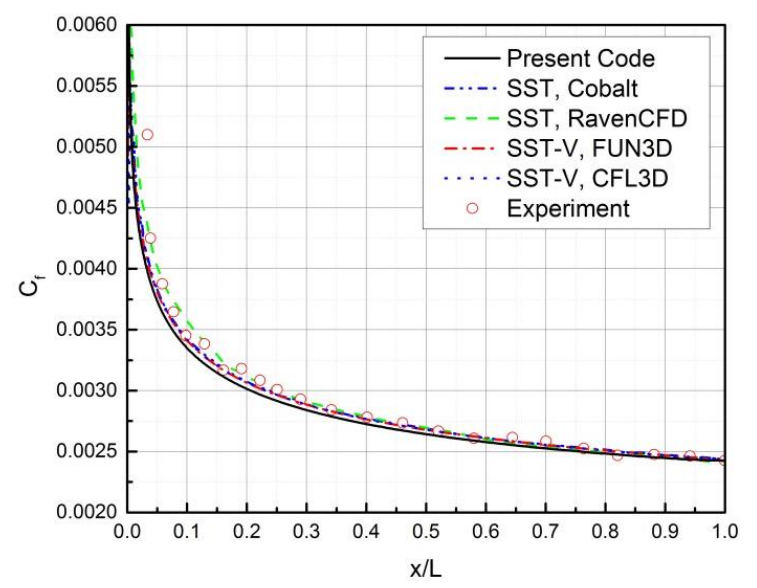

Fig. 7. Skin friction coefficient along flat plate surface.

\section{B. 2-D NACA 0012 Airfoil}

NASA Langley turbulent online resources [13] offers mainly validation data for airfoil case, so verification data are less complete; specifically, they provide results for only the 2nd finest grid, which are obtained using CFL3D, FUN3D, and from the Russian flow solver NTS for $C_{d}$ and $C_{f}$ at all angles of attack. However, Jacob A. and Roy [3] conducted a verification study for all grid levels at $\alpha=0^{\circ}$ using Cobalt and RavenCFD commercial flow solvers with Menter's SST turbulence model and other turbulence models. For code verification, Fig. 9 (a) illustrates predicted drag coefficient for NACA 0012 airfoil; Fig. 9(b) presents the comparison of the computed skin friction coefficient at the upper-surface location at $x / c=0.51, y / c=0.051$. Dashed horizontal lines mark $\pm 2 \%$ of the average results for the 2 nd finest grid from
NASA benchmark solutions. To show the more pertinent results on the finest grids, some coarse grid results are truncated from Fig. 9.

For $C_{d}$ prediction on the finest grid, our result differs from Cobalt and RavenCFD (for SST) solutions by $2.09 \%$ and by $3.6 \%$ for predicted $\mathrm{C}_{f}$. For the 2 nd finest grid, the estimated $\mathrm{C}_{d}$ differs from the benchmark values by less than $1.8 \%$ and by $2.8 \%$ from Cobalt and RavenCFD results, while $\mathrm{C}_{f}$ solution differs by less than $3.4 \%$ from those of CFL3D, Cobalt, and RavenCFD. These comparisons indicate that the predicted results are relatively close to the other commercial flow solver.

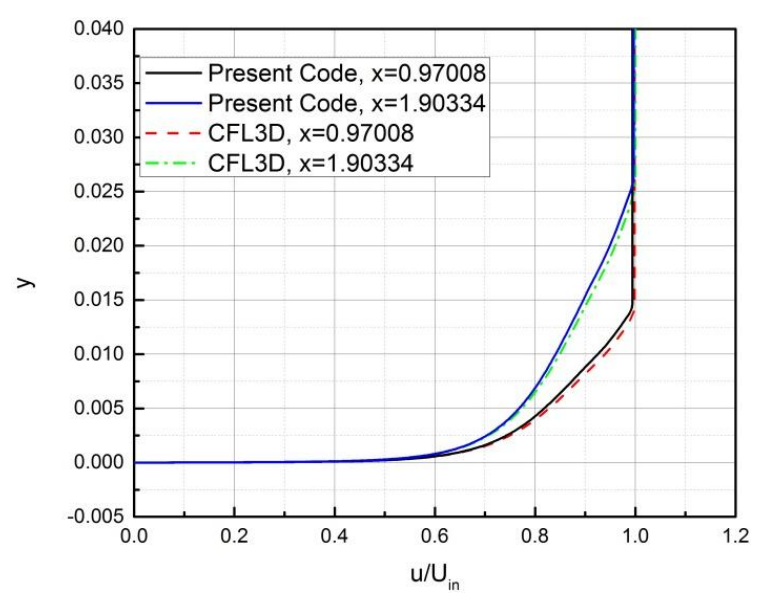

Fig. 8. Stream-wise velocity, finest grid.

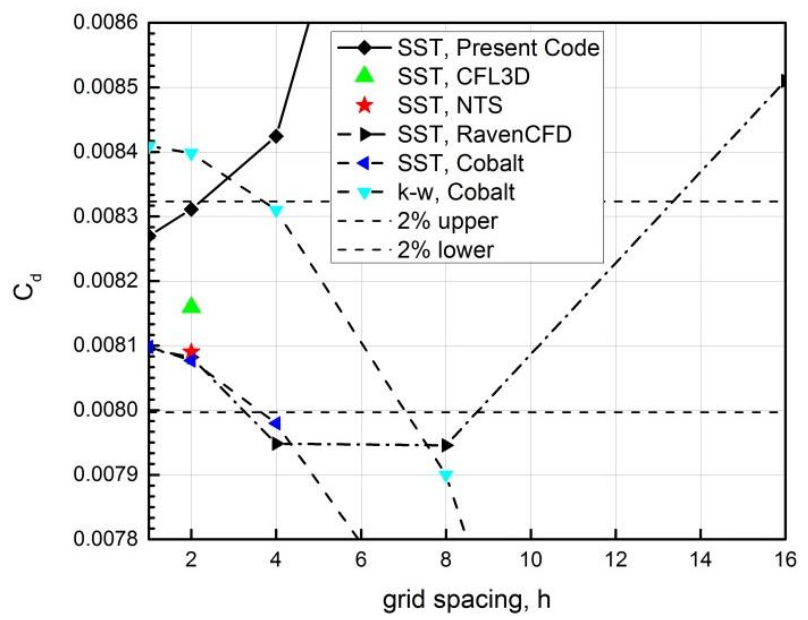

(a) Drag coefficient.

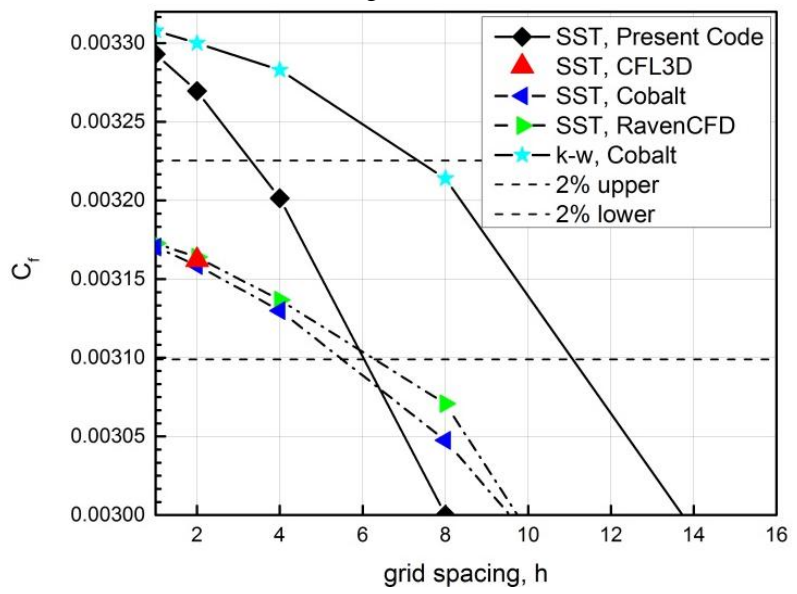

(b) Skin-friction coefficient at $x / c=0.51, y / c=0.051$.

Fig. 9. NACA 0012 airfoil, $\alpha=0^{\circ}, C_{d}$ and $C_{f}$ at $M_{\infty}=0.15$ on 5 systematically refined meshes. 
Fig. 10 compares $C_{L^{-}} \alpha$ and $C_{L^{-}} C_{d}$ with those of CFL3D, FUN3D, NTS, and experimental from [13], [31]. Good agreement can be seen between our results and the other results. Fig. 11 shows the local skin friction estimated values for the NACA 0012 at $\alpha=0^{\circ}$ for the 2nd finest grid from our code, CFL3D, Cobalt, and RavenCFD. Fig. 11(b) shows a closer view, where the scale zooms to $10 \%$ chord length. Over nearly the entire upper surface, differences among all solvers with each other are below $0.5 \%$ and below $1.0 \%$ with our code predicted results. Inside the region of transition from laminar to turbulent boundary layer the differences are significantly larger. The maximum difference between Cobalt and CFL3D is 15\%; between Cobalt and RavenCFD is $44 \%$; and $35 \%$ between RavenCFD and CFL3D. For our predicted results the maximum differences are; $15 \%$ from CFL3D; 30\% from Cobalt; and 15\% from RavenCFD. Thus, it may be generally concluded that the governing equations and the Menter's SST turbulence model are implemented correctly in our code for a non-zero pressure gradient. Table II shows observed order of accuracy as the code verification, and the total predicted uncertainty due to numerical error $\left(G C I_{\text {fine }}\right.$ and $\left.R D E_{\text {band }}\right)$ for $C_{d}$ and $C_{f}$ as the solution verification. There are no observed order and uncertainty data available for FUN3D and CFL3D to complete the comparison data.

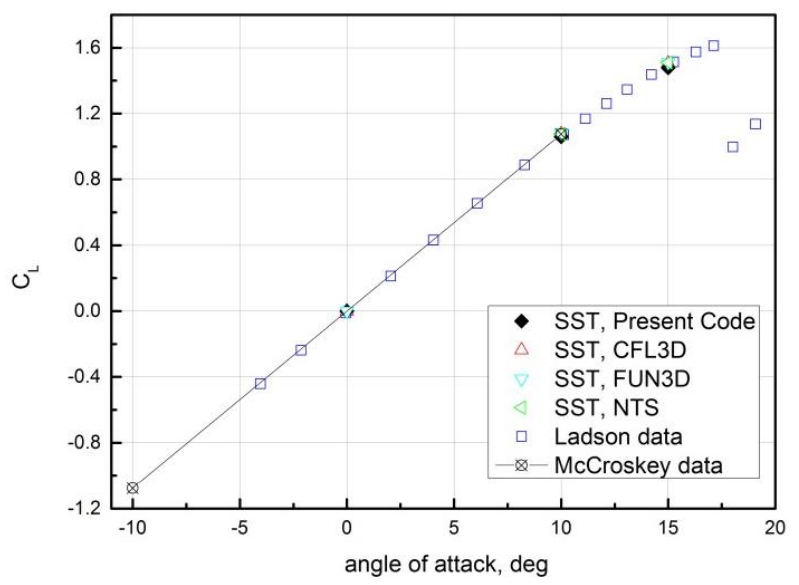

(a) $C_{L}$ vs. $\alpha$

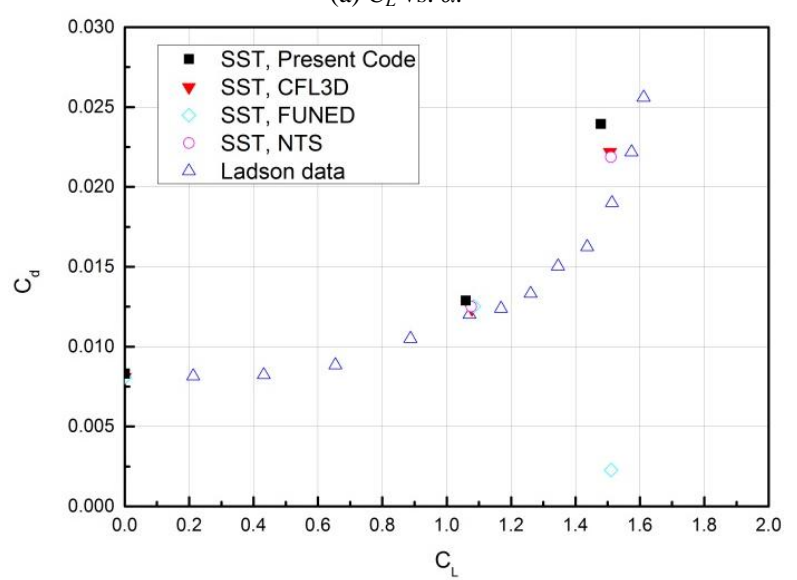

(b) $\mathrm{C}_{\mathrm{L}}$ vs. $\mathrm{C}_{\mathrm{d}}$.

Fig. 10. $C_{L}, C_{d}$ and $\alpha$ comparison over NACA 0012 airfoil at $M_{\infty}=0.15$, on 2nd finest grid.

Our code results for this case display significantly less numerical uncertainty $(\leq 0.32 \%)$ than experiment's measurement uncertainty (2.5\%) [14], while the results from
Cobalt and RavenCFD display $(\leq 0.28 \%)$ numerical uncertainty. Such solution behavior further indicates that the Menter's SST turbulence model is implemented correctly for this case.

TABLE II: NACA 0012 AIRFOIL AT ZERO ANGLE OF ATTACK

\begin{tabular}{|l|l|c|c|c|c|c|c|}
\hline \hline \multirow{2}{*}{$\begin{array}{l}\text { Turb- } \\
\text { ulence } \\
\text { model }\end{array}$} & \multirow{2}{*}{$\begin{array}{l}\text { Flow } \\
\text { solver }\end{array}$} & $P$ & $G C I_{\text {fine }} \%$ & $R D E_{\text {band }} \%$ & $\mathrm{P}$ & $G C_{I_{\text {fine }}} \%$ & $R D E_{\text {band }} \%$ \\
\cline { 3 - 9 } SST & $\begin{array}{l}\text { Present } \\
\text { Code }\end{array}$ & 3.02 & 0.23 & 0.239 & 1.57 & 0.32 & 0.31 \\
\hline & Cobalt & 2.14 & 0.11 & - & 1.70 & 0.28 & - \\
\hline & RavenCFD & 3.43 & 0.16 & - & 1.33 & 0.02 & - \\
\hline \hline
\end{tabular}

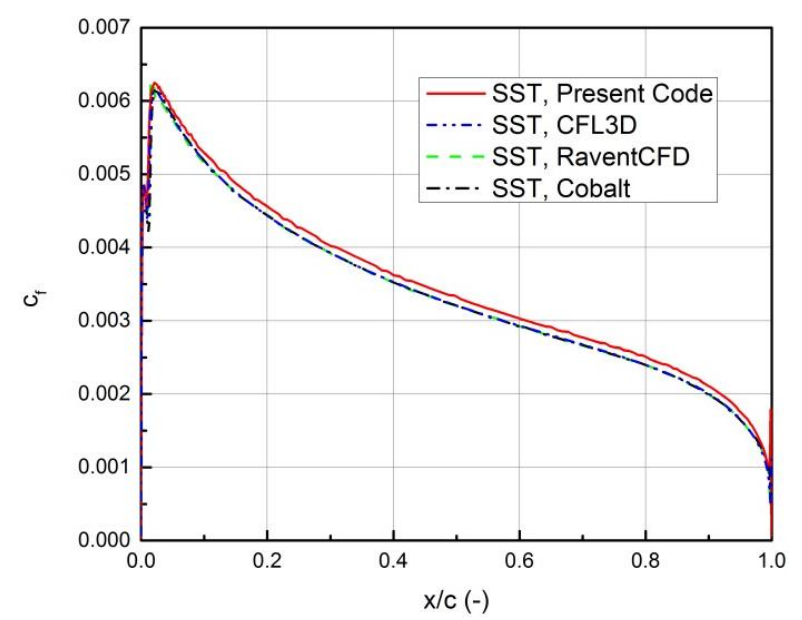

(a) Skin friction profile.

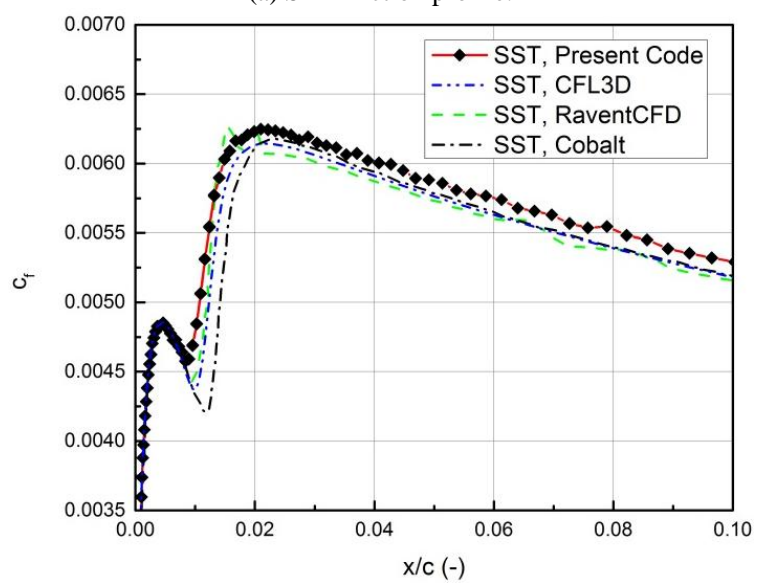

(b) Close-up view of skin friction.

Fig. 11. $\mathrm{C}_{f}$ along NACA 0012 airfoil upper surface at $\alpha=0^{\circ}$ and $M_{\infty}=0.15$, on 2 nd finest grid.

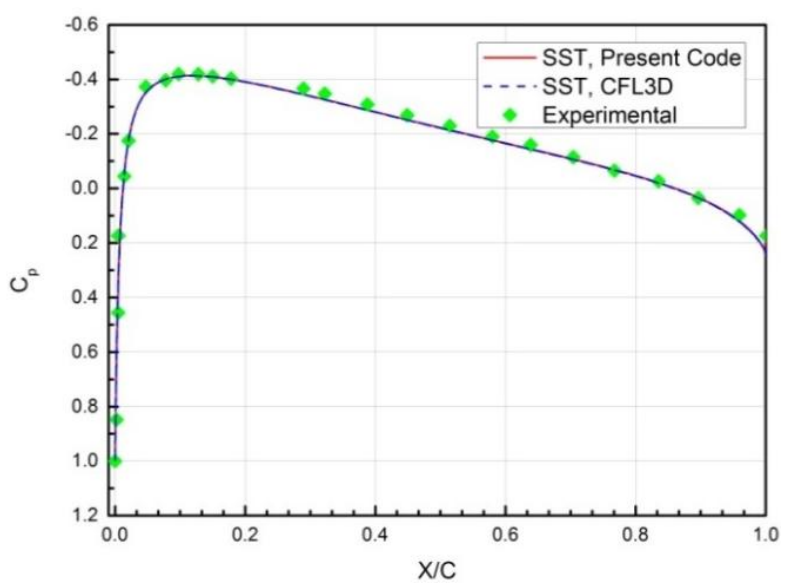

Fig. 12. Comparison of $\mathrm{C}_{p}$ for NACA 0012 airfoil at $\alpha=0^{\circ}$. 


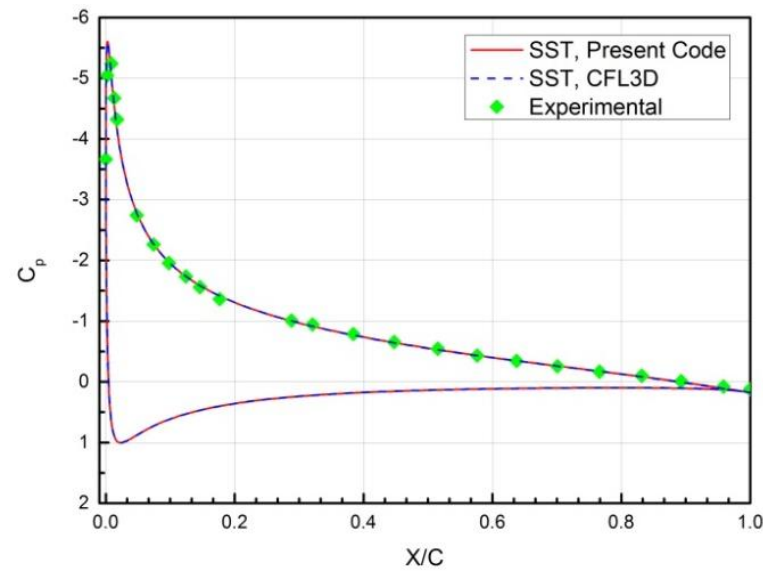

Fig. 13. Comparison of $\mathrm{C}_{p}$ for NACA 0012 airfoil at $\alpha=10^{\circ}$.

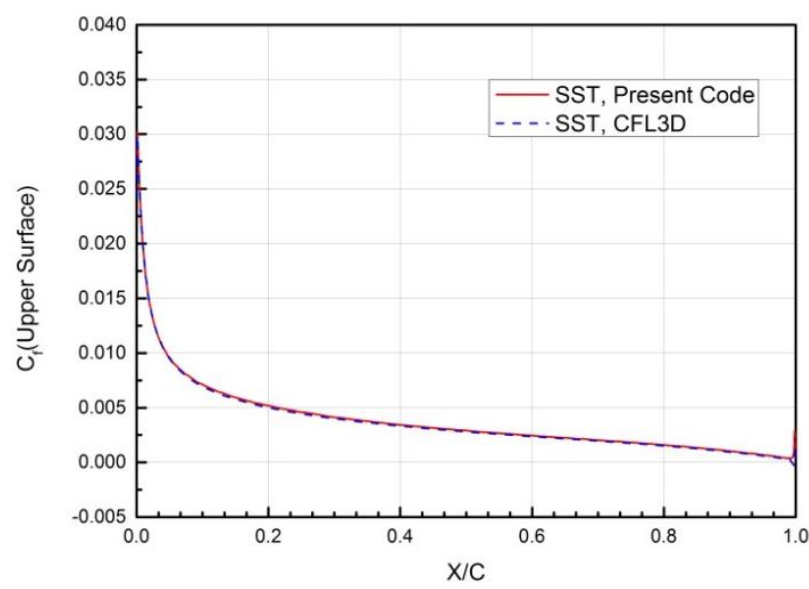

Fig. 14. Comparison of $\mathrm{C}_{f}$ for NACA 0012 airfoil at $\alpha=10^{\circ}$.

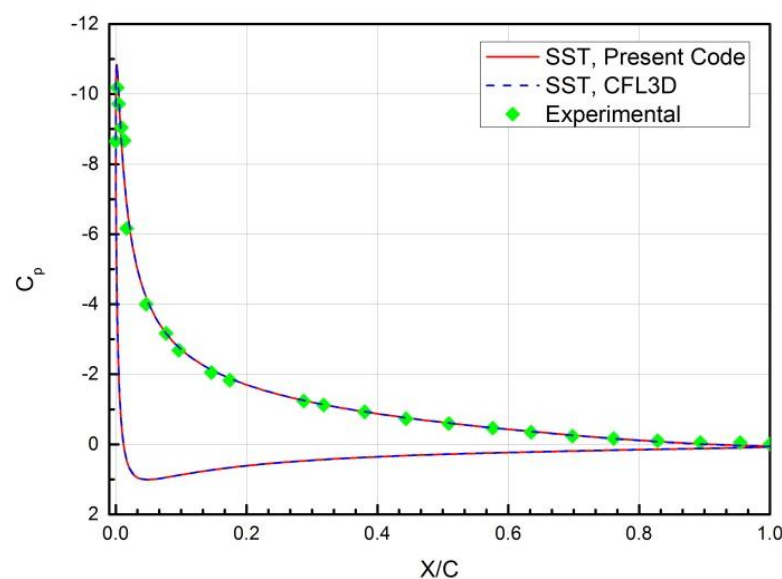

Fig. 15. Comparison of $C_{p}$ for NACA 0012 airfoil at $\alpha=15^{\circ}$.

For model validation, Figs. 12 to 16 show the comparison of $C_{p}$ and $C_{f}$ distributions at $\alpha=0^{\circ}, \alpha=10^{\circ}$, and $\alpha=15^{\circ}$ with experimental data conducted by Gregory and O'Reilly [31], respectively. The experimental data are available only for $C_{p}$. The only upper-surface distributions are shown for $C_{f}$. These distributions for $C_{p}$ are nearly coinciding with those of experimental data and CFL3D and show satisfactory accuracy of turbulent flow computations. $C_{f}$ distribution coinciding with CFL3D results, which is indicate an excellent agreement. Table III summarizes the model validation activity by comparing our results with those of CFL3D, FUN3D, NTS, Cobalt, RavenCFD, and Ladson's experimental data [14]. Our predicted results for $\alpha=0^{\circ}$ differ by $1.1 \%$ of the experimental measurements. The other solvers differ within $0.84 \%$ of the experimental measurements. At $\alpha=10^{\circ}$ the estimated $C_{d}$ differs by less $8.1 \%$ from the experiment and $C_{L}$ differs by less than $1.06 \%$. At $\alpha=15^{\circ}, C_{d}$ differs by $7.8 \%$ from CFL3D but shows highly difference from the experiment $(31 \%)$ like other solvers. $C_{L}$ gives $1.02 \%$ difference from the experiment. Thus, for the 2-D NACA 0012 airfoil at $\alpha=0^{\circ}, 10^{\circ}$, and $15^{\circ}$ the predictions of $\mathrm{C}_{d}$ and $\mathrm{C}_{L}$ match well with experiment, CFL3D, FUN3D, NTS, Cobalt, and RavenCFD.

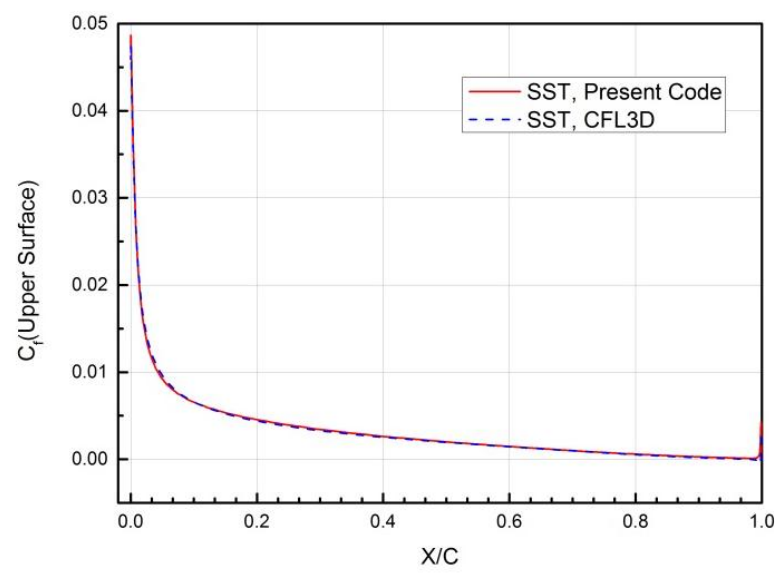

Fig. 16. Comparison of $\mathrm{C}_{f}$ for NACA 0012 airfoil at $\alpha=15^{\circ}$.

TABLE II: NACA 0012 AIRFOIL AT ALL ANGLES OF ATTACK

\begin{tabular}{|l|c|c|c|c|c|}
\hline \hline Flow solver & Grid & $c_{d}$ & Diff. (\%) & $\mathrm{C}_{\mathrm{L}}$ & Diff. (\%) \\
\hline \multicolumn{5}{|c|}{$\alpha=0^{\circ}$} \\
\hline $\begin{array}{l}\text { Experiment, } \\
\text { Ladson(1988) }\end{array}$ & - & 0.008092 & - & 0 & - \\
\hline Present Code, SST & $h_{2}$ & 0.008311 & 2.70 & 0 & - \\
\hline Cobalt, SST & $h_{1}$ & 0.008099 & 0.09 & 0 & - \\
\hline RavenCFD, SST & $h_{1}$ & 0.008096 & 0.05 & 0 & - \\
\hline CFL3D, SST & $h_{2}$ & 0.008090 & -0.02 & 0 & - \\
\hline FUN3D, SST & $h_{2}$ & 0.008160 & 0.84 & 0 & - \\
\hline NTS, SST & $h_{2}$ & 0.008090 & -0.02 & 0 & - \\
\hline \multicolumn{7}{|c|}{$\alpha=10^{\circ}$} \\
\hline $\begin{array}{l}\text { Experiment, } \\
\text { Ladson(1988) }\end{array}$ & - & 0.01191 & - & 1.058608 & - \\
\hline Present Code, SST & $h_{2}$ & 0.012883 & 8.174643 & 1.05937 & 0.071981 \\
\hline CFL3D, SST & $h_{2}$ & 0.01236 & 3.778338 & 1.0778 & 1.812947 \\
\hline FUN3D, SST & $h_{2}$ & 0.01253 & 5.205709 & 1.0840 & 2.398622 \\
\hline NTS, SST & $h_{2}$ & 0.01251 & 5.037783 & 1.0765 & 1.690144 \\
\hline \multicolumn{7}{|c|}{$\alpha=15^{\circ}$} \\
\hline $\begin{array}{l}\text { Experiment, } \\
\text { Ladson(1988) }\end{array}$ & - & 0.018313 & - & 1.4938 & - \\
\hline Present Code, SST & $h_{2}$ & 0.023940 & 30.72681 & 1.47855 & -1.02089 \\
\hline CFL3D, SST & $h_{2}$ & 0.02219 & 21.17075 & 1.5068 & 0.870264 \\
\hline FUN3D, SST & $h_{2}$ & 0.02275 & 24.22869 & 1.5109 & 1.144732 \\
\hline NTS, SST & $h_{2}$ & 0.02187 & 19.42336 & 1.5100 & 1.084483 \\
\hline \hline
\end{tabular}

\section{CONCLUSIONS}

A broad overview of verification and validation procedures for in-house codes has been provided. A steady state simulations of two benchmark cases offered by NASA Langley are performed to study CFD computer program verification and validation. A finite volume method with cell centered structured grid is used to solve Reynolds-averaged Navier-Stokes equations of the turbulent compressible flow field. The Menter's SST turbulence model and the AUSMPW scheme are implemented. Third-order three-stage 
TVD Runge-Kutta method is applied to reach the steady state time simulation.

The model presented in this work showed good agreement with NASA Langley's flow solvers (CFL3D FUN3D) [13] and with Cobalt, RavenCFD flow solvers [3], which are previously verified 2nd-order accurate for Menter's SST turbulence model. Specifically, the 2-D zero pressure gradient flat plate clearly demonstrate solutions that approach 2nd-order observed accuracy $(p=1.49)$ and numerical uncertainty $\left(\mathrm{GCI}_{\text {fine }}\right.$ and $\left.\mathrm{RDE}_{\text {band }}\right)$ is estimated to be less than $0.36 \%$. Further, skin friction coefficient results displayed good agreement with Wieghardt and Tillmann [15] experimental data for $\mathrm{x} / \mathrm{L}>0.05$, along with CFL3D, FUN3D, Cobalt and RavenCFD. Thus, one can conclude that the turbulence model has been correctly implemented for turbulent boundary layer flow.

The results for 2-D NACA 0012 airfoil case at $\alpha=0^{\circ}, 10^{\circ}$, and $15^{\circ}$ are compared with Ladson [14] experimental data, CFL3D and FUN3D for pressure coefficient, and with Cobalt and RavenCFD at $\alpha=0^{\circ}$. The skin friction coefficient results are compared with those of CFL3D, FUN3D, Cobalt, and RavenCFD: the results showed good agreement. Additionally, observed order of accuracy $(p=1.57)$ was close to the formal order of accuracy. The numerical uncertainty is predicted to be less than $0.32 \%$. Thus, it may be generally concluded that the governing equations and the Menter's SST turbulence model are implemented correctly in our code for a non-zero pressure gradient.

The presented evidences indicated that the governing equations are solved correctly and Menter's SST turbulence model is implemented correctly, and then, the code is verified and validated. This gives a practical aspect of verification and validation procedures for in-house codes.

The current code has been restricted to simulate the non-reacting turbulent flows in two-dimensional Cartesian or axisymmetric geometries. In future work, we plan to extend the present code to be able to predict the reaction phenomena taking place in the solid-fuel ramjet (SFRJ) combustion chamber.

\section{REFERENCES}

[1] W. L. Oberkampf, M. Sindir, and A. Conlisk, "Guide for the verification and validation of computational fluid dynamics simulations," AIAA Journal, January, 2002.

[2] C. J. Roy and W. L. Oberkampf, "A comprehensive framework for verification, validation, and uncertainty quantification in scientific computing," Computer Methods in Applied Mechanics and Engineering, vol. 200, pp. 2131-2144, June 2011.

[3] F. A. Jacob and J. R. Christopher, "Verification and validation of reynolds-averaged navier-stokes turbulence models for external flow," Aerospace Science and Technology, vol. 32, pp. 84-93, Jan. 2014.

[4] C. J. Roy, "Review of code and solution verification procedures for computational simulation," Journal of Computational Physics, vol. 205, pp. 131-156, May 2005.

[5] P. J. Roache, "Code verification by the method of manufactured solutions," Journal of Fluids Engineering, vol. 124, pp. 4-10, Nov. 2001.

[6] W. L. Oberkampf and F. G. Blottner, "Issues in computational fluid dynamics code verification and validation," AIAA Journal, vol. 36, pp. 687-695, May 1998.

[7] P. J. Roache and S. Steinberg, "Symbolic manipulation and computational fluid dynamics," AIAA Journal, vol. 22, pp. 1390-1394, 1984.
[8] T. G. Trucano, M. Pilch, and W. L. Oberkampf, "On the role of code comparisons in verification and validation," Sandia National Laboratories, 2003.

[9] C. J. Roy, "Review of discretization error estimators in scientific computing," AIAA Paper, Florida, Jan. 2010.

[10] O. Tong, A. Katz and D. Work, "Turbulent flow validation in the helios strand solver," in Proc. 52nd Aerospace Sciences Meeting AIAA, Scitech 2014, National Harbor, Maryland, USA, 2014.

[11] A. Hashimoto, M. Murayama, K. Yamamoto, T. Aoyama, and K. Tanaka, "Turbulent flow solver validation of FaSTAR and UPACS," in Proc. 52nd Aerospace Sciences Meeting AIAA, Scitech 2014, National Harbor, Maryland, USA, 2014.

[12] L. Cambier, V. Gleize, and J. Mayeur, "Verification and validation of the Onera elsA flow solver on RANS benchmarks," in Proc. 52nd Aerospace Sciences Meeting AIAA, 2014-0239, Scitech 2014, National Harbor, Maryland, USA, 2014.

[13] C. L. Rumsey. Turbulence modeling resource. [Online]. Available: http://turbmodels.larc.nasa.gov/

[14] C. L. Ladson, "Effects of independent variation of Mach and Reynolds numbers on the low-speed aerodynamic characteristics of the NACA 0012 airfoil section," NASA Langley Research Center, Hampton, VA, United States, 1988.

[15] K. Wieghardt and W. Tillman, On the Turbulent Friction Layer for rising Pressure, 1951

[16] J. Blazek, Computational Fluid Dynamics: Principles and Applications, 1st ed., Elsevier, 2001, ch. 2, pp. 16-17.

[17] F. R. Menter, "Two-equation eddy-viscosity turbulence models for engineering applications," AIAA Journal, vol. 32, pp. 1598-1605, August 1994.

[18] D. Wilcox, "Multiscale model for turbulent flows," in Proc. 24th AIAA Aerospace Sciences Meeting, vol. 26, pp. 1311-1320, Nov. 1986.

[19] F. Menter, "Influence of freestream values on k-omega turbulence model predictions," AIAA Journal, vol. 30, pp. 1657-1659, 1992.

[20] W. Jones and B. Launder, "The prediction of laminarization with a two-equation model of turbulence," International Journal of Heat and Mass Transfer, vol. 15, pp. 301-314, Feb. 1972.

[21] D. C. Wilcox, Turbulence Modeling for CFD, DCW industries La Canada, CA, 1998.

[22] KH. Kim, C. Kim, and O. H. Rho, "Methods for the accurate computations of hypersonic flows: I. AUSMPW+ scheme," Journal of Computational Physics, vol. 174, pp. 38-80, Nov. 2001.

[23] B. Van Leer, "Towards the ultimate conservative difference scheme. V. A second-order sequel to Godunov's method," Journal of Computational Physics, vol. 32, pp. 101-36, July 1979.

[24] G. V. Albada, B. V. Leer, and J. Roberts, "A comparative study of computational methods in cosmic gas dynamic," Astronomy and Astrophysics, vol. 108, pp. 76-84, 1982.

[25] C. W. Shu and S. Osher, "Efficient implementation of essentially non-oscillatory shock-capturing schemes," Journal of Computational Physics, vol. 77, pp. 439-471, August 1988.

[26] T. J. Poinsot and S. Lelef, "Boundary conditions for direct simulations of compressible viscous flows," Journal of Computational Physics, vol. 101, pp. 104-29, July 1992.

[27] P. R. Spalart and C. L. Rumsey, "Effective inflow conditions for turbulence models in aerodynamic calculations," AIAA Journal, vol. 45, pp. 2544-2553, Oct. 2007.

[28] L. F. Richardson and J. A. Gaunt, "The deferred approach to the limit. Part I. Single lattice. Part II. Interpenetrating lattices," Philosophical Transactions of the Royal Society of London Series A, Containing Papers of a Mathematical or Physical Character, vol. 226, pp. 299-361, 1927.

[29] L. F. Richardson, "The approximate arithmetical solution by finite differences of physical problems involving differential equations, with an application to the stresses in a masonry dam," Philosophical Transactions of the Royal Society of London Series A, Containing Papers of a Mathematical or Physical Character, vol. 210, pp. 307-357, 1911.

[30] P. J. Roache, Verification and Validation in Computational Science and Engineering, Hermosa, 1998.

[31] N. Gregory and C. OReilly, "Low-speed aerodynamic characteristics of NACA 0012 aerofoil section, including the effects of upper-surface roughness simulating hoar frost," Reports and Memoranda No. 3726, London, UK, Jan. 1970. 


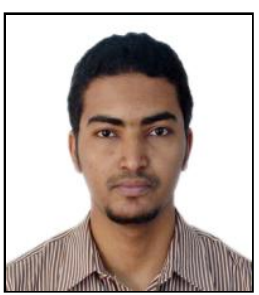

Omer Musa received his bachelor of science degree in mechanical engineering from Omdurman Islamic University, Sudan in 2008; the master of science in mechanical engineering from Sudan university of science and technology, Sudan in 2013. He has been appointed as a lecturer at Omdurman Islamic University, Khartoum, Sudan since 2009. He is currently pursuing his Ph.D. degree in mechanical engineering, Nanjing University of Science and Technology, China. His research interests include computational fluid dynamics.

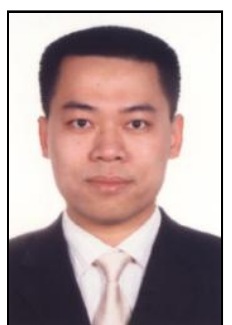

Chen Xiong received his bachelor of engineering degree in mechanical engineering from Nanjing university of science and technology, China in 2000 his Ph.D. degree in mechanical engineering, Nanjing university of science and technology, China in 2005 Dr. Chen is holding a position of associate professor and an associate dean of School of Mechanical Engineering, Nanjing University of Science and Technology, China. His research interests include computational fluid dynamics. 